\title{
Fast fatigue limit estimation of woven composite materials by self-heating analysis
}

\author{
by G. Bai*, B. Lamboul*, P. Paulmier*, M. Kaminski*, J.-M. Roche* and S. Baste** \\ * ONERA, Composite Materials and Structures Dept., 92322 Châtillon cedex, France. gabriele.bai@onera.fr \\ ** Univ. of Bordeaux, I2M, UMR 5295, F-33400 Talence, France.
}

\begin{abstract}
The fatigue limit of materials is usually difficult to estimate, and requires an expensive experimental campaign to test every sample under a large number of cycles in order to determine the asymptote of the S-N curve. An experimental study is here reported with the purpose to show an efficient and fast way to estimate the fatigue limit of a woven composite using only one sample. The studied material is subjected to stepped fatigue cycles, for increasing values of stress, with a fixed frequency and a constant stress ratio. The surface temperature variation and its heating rate are analyzed.
\end{abstract}

\section{Nomenclature}

$\begin{array}{cl}\theta & \text { Sample heating } \\ T & \text { Sample temperature } \\ T_{U} & \text { Upper clamp temperature } \\ T_{L} & \text { Lower clamp temperature } \\ \bar{\theta} & \text { Stabilised heating } \\ \dot{\theta} & \text { Initial heating rate }\end{array}$

$\begin{array}{ccl}K & f & \text { Excitation frequency } \\ K & R & \text { Stress ratio } \\ K & t & \text { Time } \\ K & \rho & \text { Density } \\ K & C_{P} & \text { Specific heat } \\ m K / s & \Phi & \text { Mechanical dissipation }\end{array}$

$H z$
-
$s$
$\mathrm{~kg} / \mathrm{m}^{3}$
$\mathrm{~J} / \mathrm{kg} / \mathrm{K}$
$\mathrm{J} / \mathrm{m}^{3}$

\section{Introduction}

The fatigue of materials is the progressive structural damage that occurs when a material is subjected to cyclic loading. As a consequence, the material can fail even if the operating stress is lower than its tensile stress limit. The stress value below which failure probability is almost zero, even for an infinite number of loading cycles, is named fatigue limit (or endurance limit) [1]. Experimentally, the fatigue limit of a material is estimated by performing several fatigue tests with very high numbers of cycles, using standardised samples. Each specimen is periodically loaded around a constant stress value (different for each sample) in order to determine the number of cycles to failure. The results are then reported in a graph, called the S-N graph, which represents the evolution of the failure number of cycles with the excitation stress. Theoretically, the horizontal asymptote of the curve represents the fatigue limit of the material [2]. This strategy has the disadvantage of requiring numerous time-consuming mechanical tests, as long as a large number of specimens, in order to properly estimate the entire S-N.

Over the last few years, to find a solution to this problem, some authors have proposed an empirical method based on the analysis of self-heating to determine the average endurance limit of materials $[3,4,5]$. A self-heating test consists in applying to a sample a reduced number of fatigue cycles (no more than a few thousands), for increasing values of stress, and to monitor the evolution of its temperature. It is generally observed that after a certain level of loading, theoretically close to the average endurance limit of the material, the stabilised temperature significantly increases, which is due to the creation and propagation of damage in the material, as a consequence of the fatigue loading. This stress value is a first estimation of the fatigue limit; at the very least, it provides key information to the aeronautical industry for fatigue dimensioning of composite structures.

The main advantage of this approach is the gain of time it enables: not only is the number of dynamic cycles drastically reduced, but also does the whole experimental campaign only require one specimen. This fast method has been studied on various materials, such as metal alloys $[3,4,6]$, elastomers $[7,8]$ and more recently on short and long fiber composites $[9,10]$.

In this context, this work aims at investigating the relevance of this method for carbon/epoxy woven composite materials. The present article shows that not only the stabilised temperature, but also the initial heating rate of the sample can be used to estimate its endurance limit. Two different material configurations are tested in order to study the influence of the thickness on the fatigue behavior of this kind of material. First conclusions are discussed in the final part of the paper. 


\section{Materials and test configuration}

The studied material is a carbon/epoxy 2D woven composite. Four samples $\left(250 \times 25 \mathrm{~mm}^{2}\right)$ are tested: the first two are constituted of 8 plies (thickness of $3.72 \mathrm{~mm}$ ) and the other two of 16 plies (thickness of $7.44 \mathrm{~mm}$ ). Each ply is made of weaving carbon fibers in a five-harness satin configuration, as illustrated in figure 1. All four tested samples present $\mathrm{a} 90^{\circ}$ orientation compared to the warp direction.
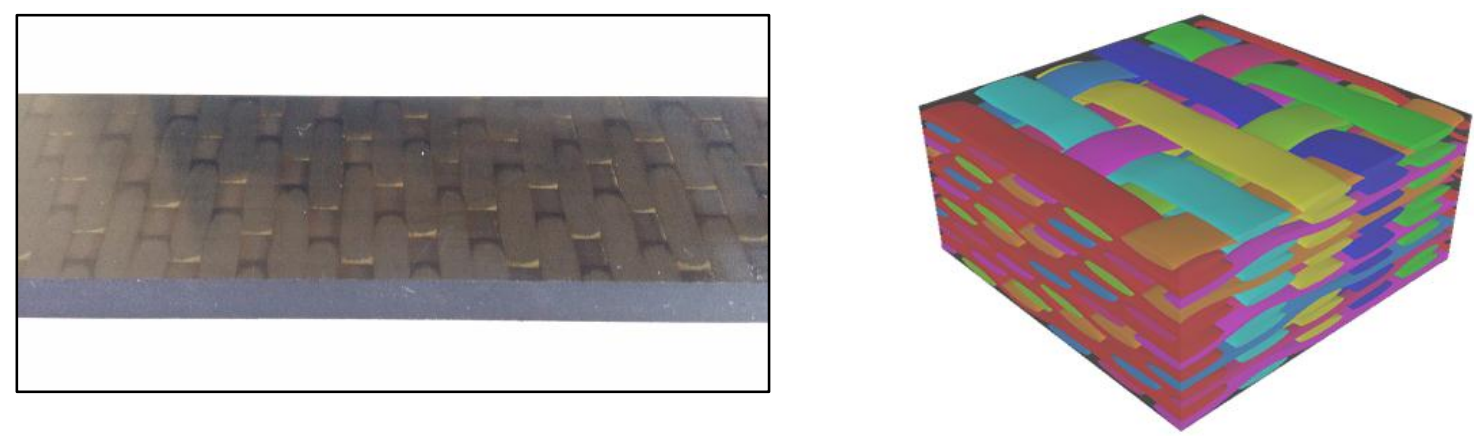

Fig. 1. Five-harness satin weaved composite. Left: Close-up picture of a sample. Right: Five-harness satin configuration generated by TexGen software.

Each sample is subjected to stepped fatigue cycles, with increasing values of stress (Figure 2). The stress ratio $R$ (ratio between the minimum and the maximum stresses) is fixed at 0.5 for all steps in order to limit the excitation amplitude for high stress steps: this value guarantees a number of 10 to 15 steps. The frequency also remains constant during the whole test, equal to $5 \mathrm{~Hz}$, which is a satisfactory compromise between the test duration that should be as short as possible and the need to limit the heating of the sample. During each step, the heating of the sample and of the clamps is acquired by an infrared camera (FLIR X6540sc, with an acquisition frequency of $25 \mathrm{~Hz}$ ). Due to the reflecting nature of the analysed surfaces, the sample and parts of the clamps are painted with a black coating.

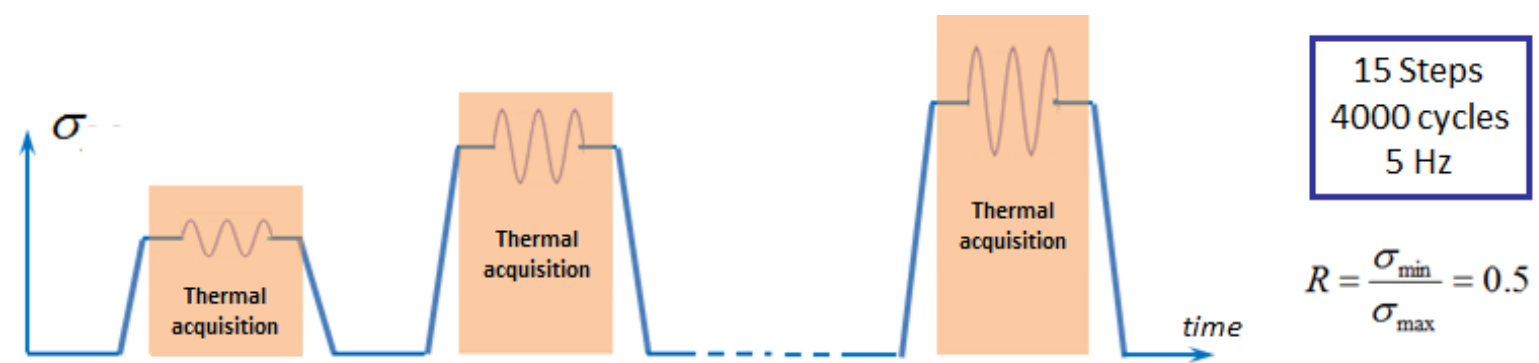

Fig. 2. Stepped self-heating test configuration.

\section{Results}

The first operation that must be done is the correction of the temperature evolution in the sample by taking into account the temperature variations in the clamps of the testing machine. As reported in figure 3 , the clamps present a temperature variation that is a consequence of the heating of the oil flow in the clamping system. In a first approximation, the clamps can be considered as thermal sources that introduce a constant thermal gradient in the sample, leading to the following expression for the sample heating:

$$
\theta(t)=T(t)-T(0)-\left[\frac{T_{U}(t)+T_{L}(t)}{2}-\frac{T_{U}(0)+T_{L}(0)}{2}\right]
$$

where $\theta$ is the heating the sample, $T$ is the sample temperature, $T_{U}$ and $T_{L}$ are the temperature of the upper clamp and of the lower clamp, respectively. 

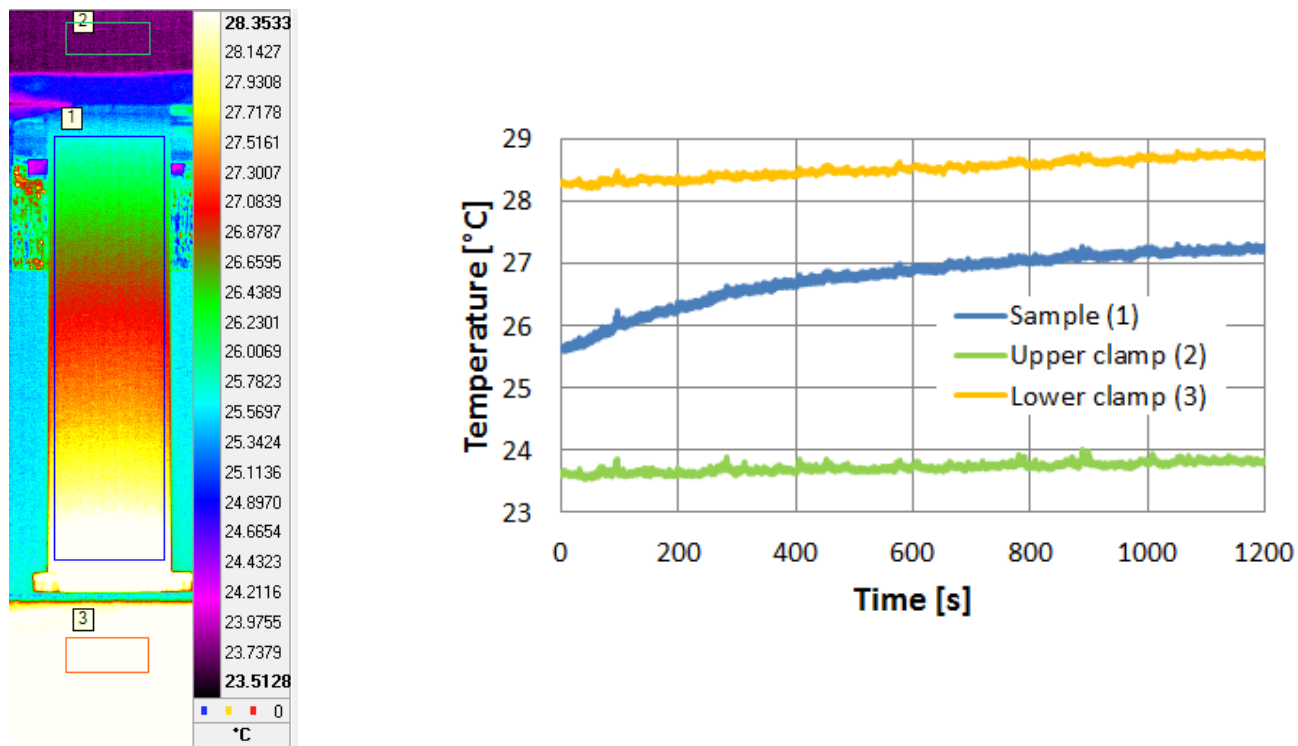

Fig. 3. Left: Raw thermal image of the sample and location of the studied zones: sample (1), upper clamp (2) and lower clamp (3). Right: Temperature variation of these zones during one step

In figure 4 an example of the corrected heating of a sample after this operation is shown for a generic stepped self-heating test. Two parameters are taken into account: the stabilised heating $\bar{\theta}$, defined as the final asymptotic value reached by $\theta$, and the initial heating rate $\dot{\theta}$, defined as the initial slope of the curve. The results for each material configuration are reported in the next paragraphs.

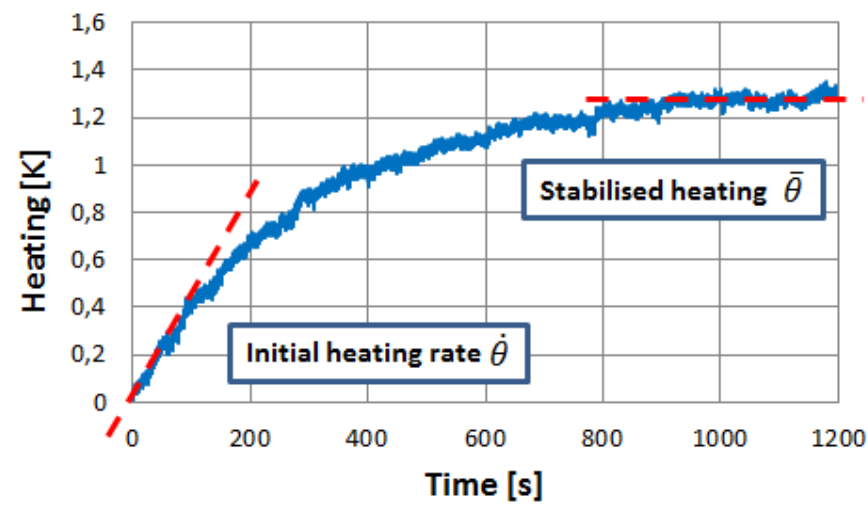

Fig. 4. Corrected heating of the sample and definition of two parameters: initial heating rate and stabilised heating.

\subsection{6 ply woven composite $(7.44 \mathrm{~mm})$}

For the 16 ply composite two different test configurations are carried out, as reported in table 1 . The first test is composed of 11 steps of 4,000 cycles $(R=0.5, f=5 \mathrm{~Hz})$ followed by 4 steps of 6,000 cycles to allow the sample to reach the stabilised temperature for a total of 68,000 cycles. The sample did not fail during test. The second sample is loaded with only 9 steps for a total of 36,000 cycles.

Table 1. Test configurations for the 16 ply samples

\begin{tabular}{|c|c|c|c|c|}
\hline & \# Steps & \# Cycles/Step & $R$ & $f[\mathrm{~Hz}]$ \\
\hline Sample 1 & $11+4$ & $4,000+6,000$ & 0.5 & 5 \\
\hline Sample 2 & 9 & 4,000 & 0.5 & 5 \\
\hline
\end{tabular}


For each step of each sample the correction procedure is applied and the stabilised temperature and the initial heating rate are assessed. In figure 5 and figure 6 are reported the variations of these two parameters with the mean stress of each step. The observed change of heating behaviour is manifested by a clear change of slope. The intersection of the two linear interpolations represents the endurance limit for the material.
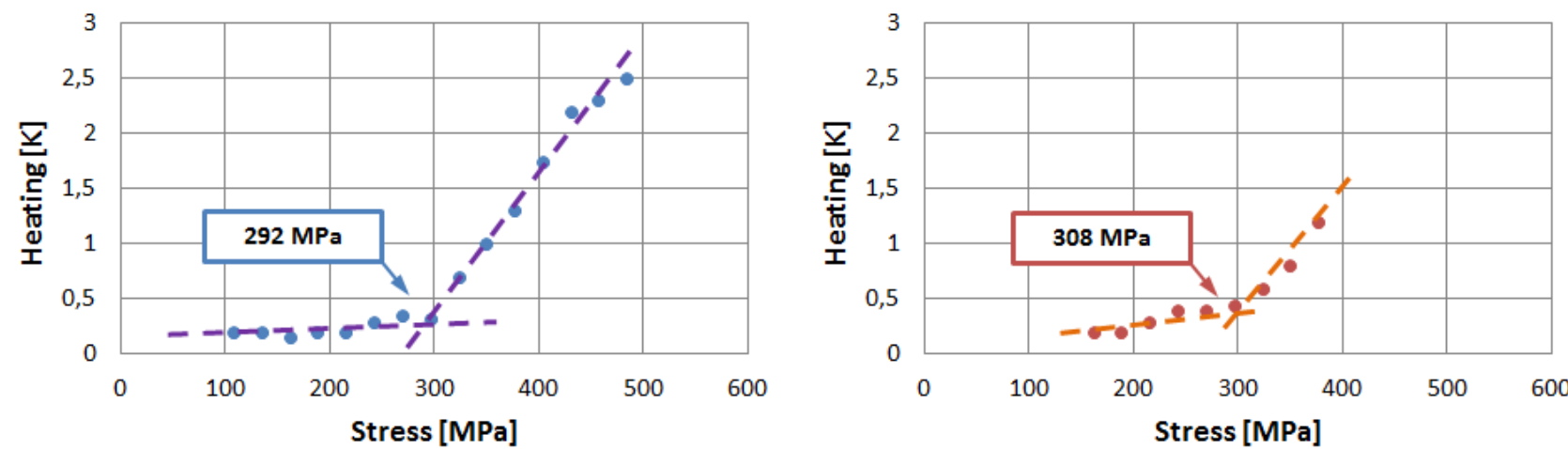

Fig. 5. Variation of heating with the step mean stress and estimation of the fatigue limit by linear interpolation of the two segments. Left: sample 1. Right: sample 2.
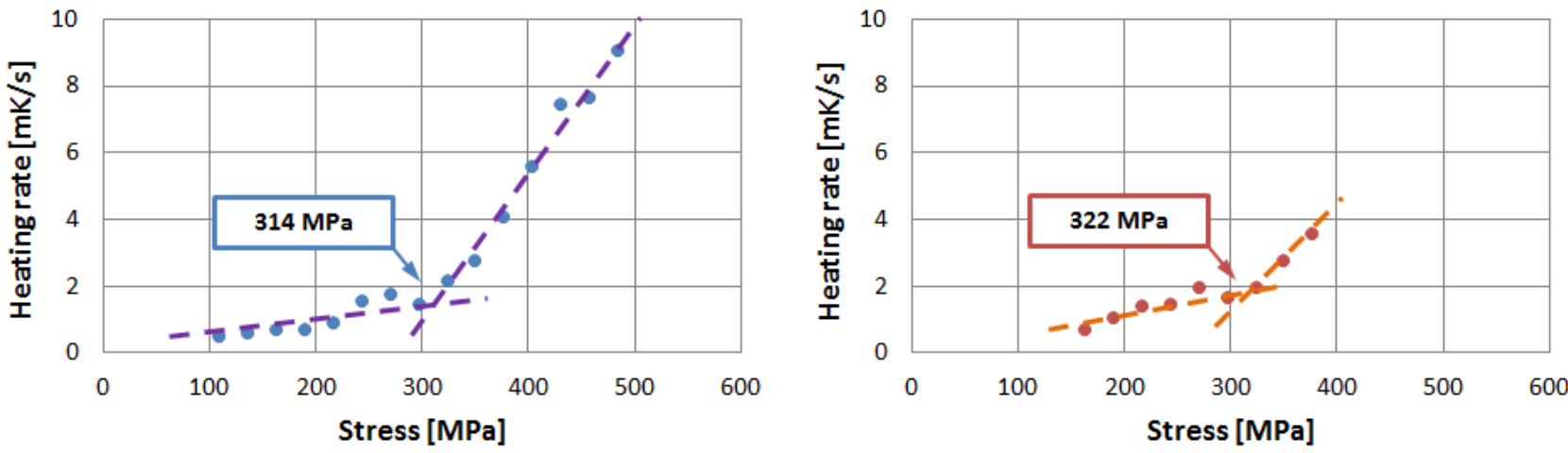

Fig. 6. Variation of initial heating rate with the step mean stress and estimation of the fatigue limit by linear interpolation. Left: sample 1. Right: sample 2.

Two important observations can be made. The first one is that there is no an important dispersion in the results obtained between the two samples (the found values agree within a $\pm 5 \%$ tolerance). Even if the test configurations are different in terms of steps and cycle numbers, the endurance limits estimated for both samples are very close to each other.

The second observation is that it is possible to estimate the fatigue limit using the initial heating rate. This means that it is not necessary to wait for the material to reach a stabilised temperature, but it is sufficient to analyse the initial variation of temperature, considerably reducing the test duration.

In order to interpret this result, a simple model can be proposed. For a generic material, with the hypothesis of external thermal sources, thermal transfers and thermoelastic effects negligible, the thermodynamics equation can be simplified into [11]:

$$
\rho C_{P} \dot{\theta}=\Phi=A * f
$$

where $\rho$ is the material density, $C_{P}$ is the specific heat, $\dot{\theta}$ is the heating rate, $\Phi$ is the mechanical dissipation that is basically the product of the volumic mechanical energy $A$ and the excitation frequency $f$.

This mechanical dissipated energy can be estimated by the analysis of the hysteresis loops in the $\sigma, \varepsilon$ graph by assessing their areas, as shown in figure 7 (left). In figure 7 (right) is reported the comparison between the mechanical energy and the thermal dissipation estimated using the initial heating rate for the sample 1. As expected, the good correlation between the two curves means that, at the beginning of the excitation, where the losses can be neglected, most of the mechanical dissipated energy is converted into a temperature rise of the sample $\dot{\theta}$. Furthermore it proves that the procedure aiming at taking into account the thermal gradient between the clamps indeed corrects the temperature increase due to external sources. 

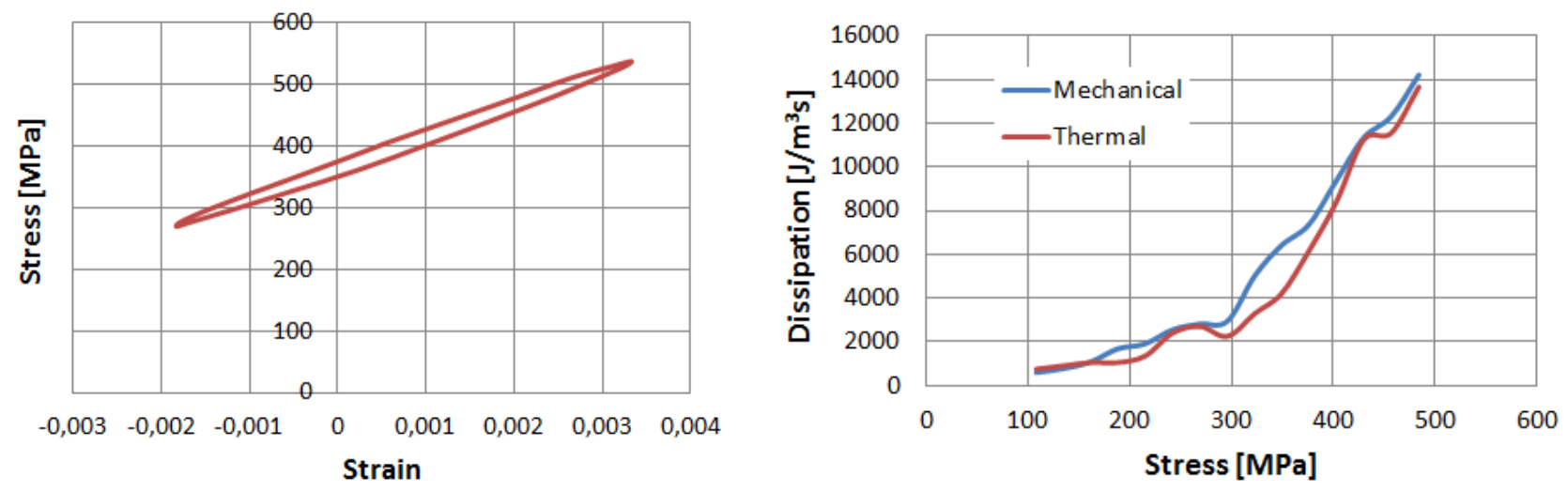

Fig. 7. Left: Hysteresis loop area estimation for sample 1 (Cycle number $\# 1,000$ for the 400 MPa mean stress step). Right: Comparison between mechanical (blue) and thermal dissipated energies (red) for sample 1.

\subsection{8 ply woven composite $(3.72 \mathrm{~mm})$}

The test configuration for the 8 ply samples is reported in table 2 and consists in a series of 7 steps of 4,000 cycles followed by 4 steps of 6,000 cycles (to reach the stabilised temperature):

Table 2. Test configurations for the 8 ply samples

\begin{tabular}{|c|c|c|c|c|}
\hline & \# Steps & \# Cycles/Step & $R$ & $f[\mathrm{~Hz}]$ \\
\hline Sample 1 & $7+4$ & $4,000+6,000$ & 0.5 & 5 \\
\hline Sample 2 & $7+4$ & $4,000+6,000$ & 0.5 & 5 \\
\hline
\end{tabular}

As for the previous samples, the correction procedure for the heating estimation is applied, and the stabilised heating and initial heating rate are assessed for each step, as shown in figure 8 and 9.
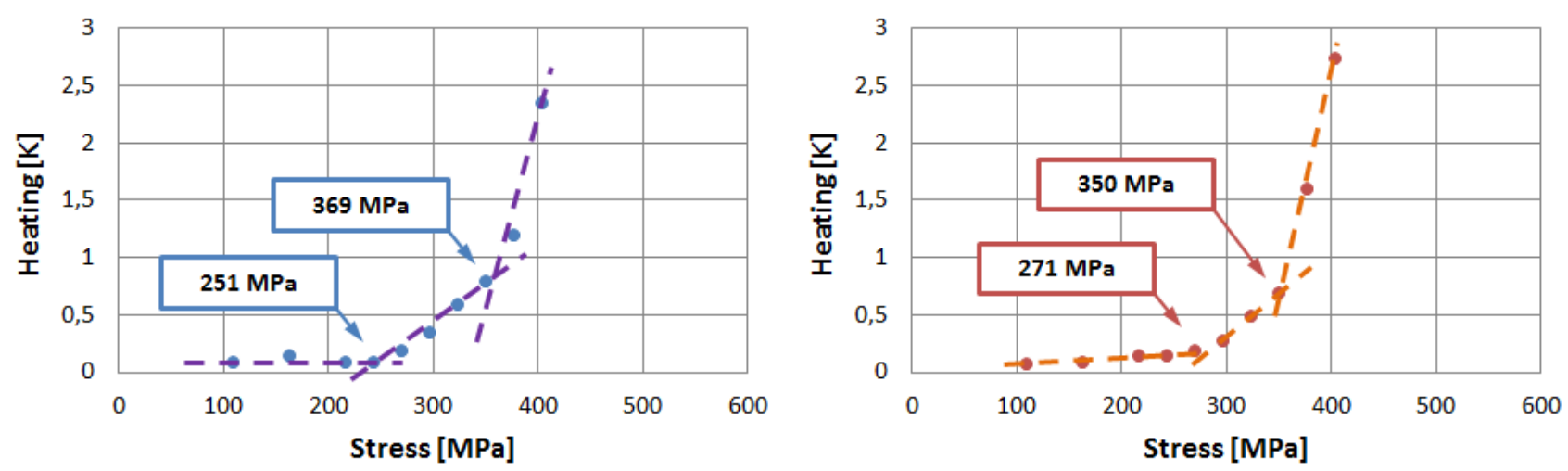

Fig. 8. Variation of heating with the step mean stress and estimation of the fatigue limit by linear interpolation for the 8 ply composite. Left: sample 1. Right: sample 2. 

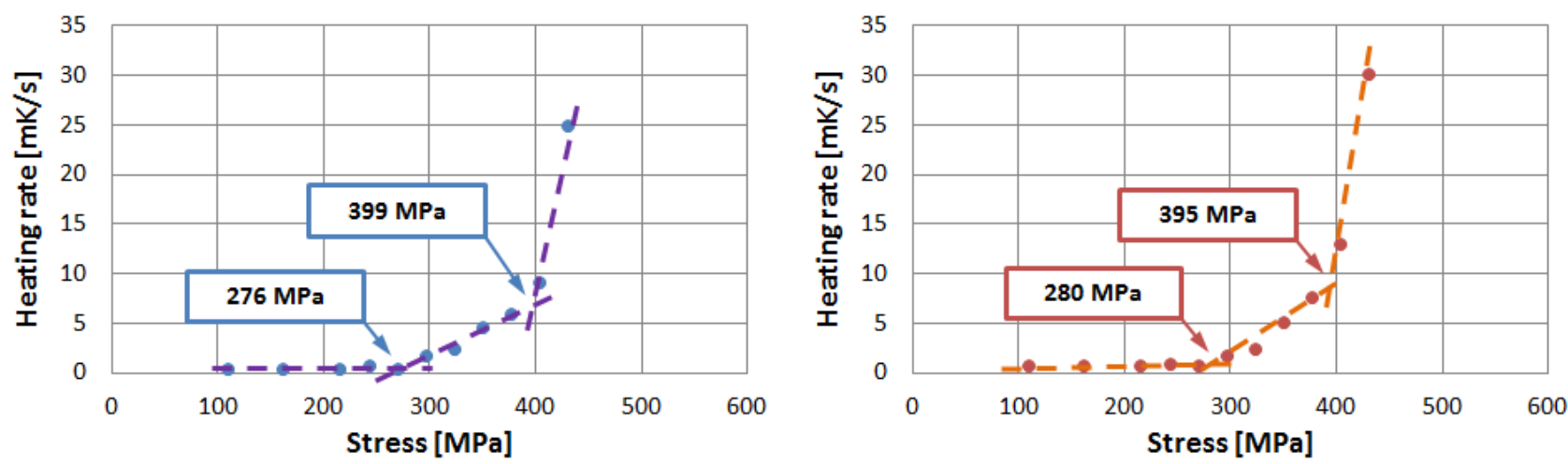

Fig. 9. Variation of initial heating rate with the step mean stress and estimation of the fatigue limit by linear interpolation for the 8 ply composite. Left: sample 1. Right: sample 2.

Unlike the 16 ply samples, the material presents a double change in the slope curves. This might indicate that there are two different damage mechanisms successively occurring in the 8 ply samples. The first slope change (around 250/300 MPa) occurs at a very close stress value for all the tested thicknesses, even if it is consistently lower for the 8 ply samples. This brings to the conclusion that the beginning of the fatigue damage seems to be the same independently of the sample thickness. The second change (around 350/400 MPa) is a characteristic of the 8 ply sample, which would mean that the thickness influences the evolution of the damage. In fact, the steps of the 8 ply samples after the second slope change introduce a macro-damage in the material (delamination and fibres ruptures can be clearly observed in the instantaneous temperature fields shown in figure 10) that brings it to failure at the beginning of the last step.
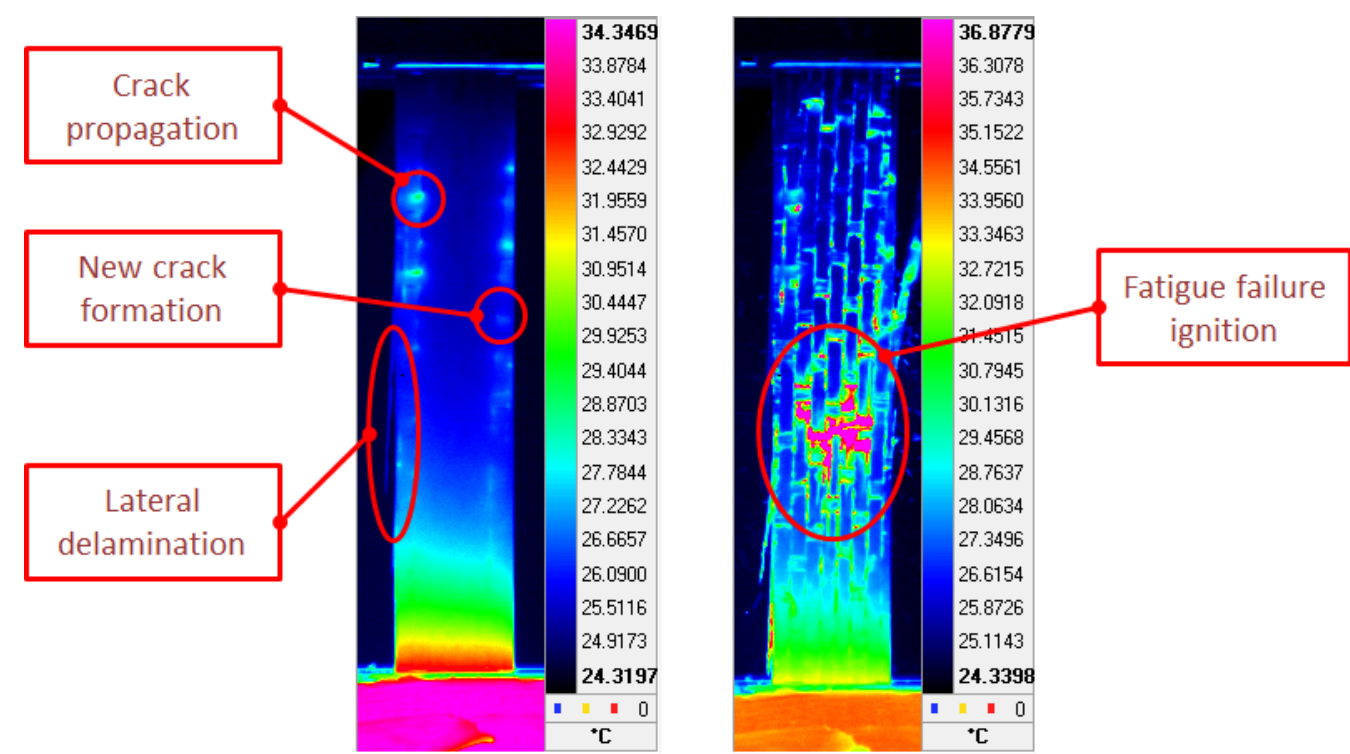

Fig. 10. Instantaneous temperature images of sample 1 (8 ply, 430 MPa mean stress step). Left: macro-damage propagation. Right: Fatigue failure of the sample.

\section{Conclusions}

The aim of this paper was to demonstrate that the empirical method to estimate a fatigue limit using thermography could be applied to woven composite materials and to assess how the thickness of samples could influence this estimation. In particular this work focused on improving this method by introducing the analysis of the initial heating rate. The results presented show that the limits estimated by measuring the final stabilised temperature for each 
step and the initial heating rate are very close. This makes it possible to reduce the test duration and data analysis. This result is checked using thermodynamics considerations and verified by mechanical analysis using the hysteresis loops.

The tests are done on samples of two different thicknesses. The results comparison brings to the conclusion that the thickness does not seem to significantly influence the fatigue limit estimation because the beginning of the fatigue damage in the material occurs at about the same excitation stress for all the samples. The thickness seems to only influence the evolution of the damage in the material. In fact, the thinner samples present a fast increase of the crack propagation that quickly merges into macro-damage, which brings the sample to fail during fatigue cycling.

This predicted limit can be used as a material parameter by engineers to optimise structure that are submitted to fatigue sollicitations. The presented method indeed guarantees that under this stress level, only a slow generation and propagation of fatigue damage should occur during operation life of structures.

As a perspective, other excitation configurations should now be analysed.

\section{REFERENCES}

[1] Norman E. Dowlings, Mechanical behavior of materials, Englewood Cliffs (NJ), Prentice Hall, 1993,780 p.

[2] Ellying, "Fatigue damage, crack growth and life prediction", Springer edition, 1997.

[3] Luong, "Infrared thermographic scanning of fatigue in metals", Nuclear Engineering and Design, Volume 158, pp 363-376.

[4] Krapez, Pacou, Gardette, "Lock-in thermography and fatigue limit of metals", Proceedings of Qirt, pp 277-282.

[5] La Rosa, Risitano, "Thermographic methodology for rapid determination of the fatigue limit of materials and mechanical components." International Journal of Fatigue 21, pp 65-73.

[6] Giancane, Panella, Dattoma, Watrisse, "Deformation and dissipated energy for high cycle fatigue of 2024-T3 aluminium alloy", Theoretical and applied fracture mechanics, Volume 2, pp 117-121.

[7] Le Saux, "An energetic criterion for the fatigue of rubbers: an approach based on a heat build-up protocol and microtomography measurements" Procedia Engineering, Volume 2, pp 949-958.

[8] Masquelier, Marco, Le Saux, Calloch, Charrier, "Thermal measurements on elastomeric materials: from the characterisation of the dissipation gradients to the prediction of the fatigue properties", Procedia Engineering 66, pp 661-668.

[9] Jegou, Marco, Le Saux, Calloch, "Fast prediction of the Wohler curve from heat build-up measurements on short fiber renforced plastic". International journal of fatigue, Volume 47, pp 259-267.

[10] Gornet, Westphal, Rozycki, Stainier, Kemlin, "Rapid determination of fatigue properties of carbon fiber epoxy matrix composite laminates by self-heating tests", ECCM16, European conference of composite materials.

[11] Lemaitre, Chaboche, "Mechanics of solids materials", Cambridge university press. 